\title{
Comunicación
}

\section{Eficacia in vitro de Morinda citrifolia L para el Control de Rhipicephalus (Boophilus) microplus (Acari: Ixodidae)}

\author{
IN VItro EfFicacy of Morinda citrifolia L IN THE CONTROL OF Rhipicephalus \\ (Boophilus) microplus (ACARI: IXODIDAE) \\ Darién Nápoles V. ${ }^{1}$, Kenia Milagro Sebasco R.², Manuel Colas C. ${ }^{3}$, \\ Wilfredo López S. ${ }^{3}$, Teresa Meireles R. ${ }^{3}$
}

\section{Resumen}

El estudio tuvo como objetivo la evaluación de la eficacia in vitro de Morinda citrifolia $\mathrm{L}$, en comparación con productos químicos convencionales (Supatraz ${ }^{\circledR} /$ amitraz y Ticomín ${ }^{\circledR} /$ cipermetrina) sobre teleoginas de Rhipicephalus (Boophilus) microplus. Las teologinas fueron recolectadas de bovinos exentos de garrapaticidas químicos. Se seleccionaron 10 garrapatas por cada placa Petri $(n=24)$ distribuidas en 8 tratamientos con 3 réplicas cada uno. Los tratamientos T1 a T5 recibieron soluciones hidroalcohólicas de $M$. citrifolia L al 10, 5, 2.5, 1.25 y $0.625 \%$, respectivamente; T6 recibió Supatraz $\mathbb{R} /$ amitraz y T7 Ticomín ${ }^{\circledR} /$ cipermetrina en diluciones de 1:1000; y T8 fue el control negativo (agua destilada), en volúmenes de $15 \mathrm{ml}$ por espacio de $5 \mathrm{~min}$. Las lecturas se hicieron a los 15 min y 24 h posexposición y se contaron las teleoginas muertas para evaluar la eficacia de los tratamientos. Los resultados indicaron una eficacia de 89.6 y $41.3 \%$ para los tratamientos químicos T6 y T7. La máxima eficacia de $M$. citrifolia L fue de $20.6 \%$ en la concentración de $10 \%$.

Palabras clave: Rhipicephalus (Boophilus) microplus, teleogina, Morinda citrifolia L, eficacia

\footnotetext{
${ }^{1}$ Universidad de Ciencias Médicas de La Habana, Cuba

${ }^{2}$ Universidad de La Habana, Cuba

${ }^{3}$ Universidad Agraria de la Habana, Mayabeque, San José, Cuba

${ }^{4}$ E-mail:darinv@infomed.sld.cu
} 
The aim of this study was to evaluate the in vitro efficacy of Morinda citrifolia $\mathrm{L}$ in comparison with conventional chemical products (Supatraz ${ }^{\circledR} /$ amitraz and Ticomín ${ }^{\circledR} /$ cipermetrina) on engorged females of Rhipicephalus (Boophilus) microplus. The adult ticks were collected from cattle free of chemical acaricides. Ten ticks were selected per Petri dish $(\mathrm{n}=24)$ and distributed in 8 treatments with 3 replicas each one. Treatments T1 to T5 received hidroalcoholic solutions of Morinda citrifolia L at 10, 5, 2.5, 1.25 y $0.625 \%$

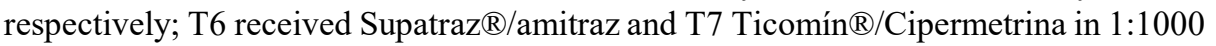
dilution; and T8 was the negative control (distilled water) in volumes of $15 \mathrm{ml}$ per Petri dish for $5 \mathrm{~min}$. The readings were carried out at $15 \mathrm{~min}$ and $24 \mathrm{~h}$ postexposition. Dead ticks were counted to determine the efficacy of the treatments. The results showed an efficacy of 89.6 and $41.3 \%$ for the chemical treatments T6 and T7, whereas the maximum efficacy for $M$. citrifolia $\mathrm{L}$ was 20.6 in the concentration of $10 \%$.

Key words: Rhipicephalus (Boophilus) microplus, teleoginas, Morinda citrifolia L, efficacy

\section{INTRODUCCIÓN}

Rhipicephalus (Boophilus) microplus es la garrapata de mayor frecuencia e importancia en la industria ganadera (Rodríguez et al., 2005) por su amplia distribución mundial (Taylor et al., 2007) y con gran capacidad de adaptación y propagación (Estrada y Venzal, 2006). Ocasiona grandes pérdidas económicas a la ganadería por la reducción de la producción de leche y carne y transmisión de agentes patógenos (virus, bacterias, rickettsias y protozoos), así como incremento en los costos para su control (Guglielmone et al., 2007).

Se disponen de métodos acaricidas químicos y no químicos para el control de las garrapatas, siendo el químico el más utilizado; entre ellos, destacan las familias de los organofosforados $(\mathrm{OF})$, piretroides sintéticos (PS), amidinas (Am), fenilpirazolonas (FP) y los endectocidas, denominados lactonas macrocíclicas (LM) (Alonso et al., 2006; Rodríguez et al., 2006a, 2010). Sin embargo, el uso irracional de estos compuestos han generado resistencia en los ácaros (Rodríguez et al., 2006b, 2007), lo que acarrea la ineficacia del producto y la necesidad de utilizar otros principios activos (FAO, 2003; Guglielmone et al., 2007). Este fenómeno determina que se requiera invertir en el desarrollo de nuevos acaricidas, así como en la búsqueda de tratamientos alternativos como los productos no químicos naturales y fitoterápicos (Ojeda et al., 2011).

Hay un interés global por investigar la presencia de principios farmacológicos en plantas, que son reconocidas como antiparasitarias y que son utilizadas por los campesinos (Bermúdez y Velásquez, 2002; Gil et al., 2006). Algunos de estos productos son eficaces, de bajo costo y no causan daño al ecosistema (Fernández y Freitas, 2007). En Cuba, la aplicación de extractos de plantas contra ectoparásitos se realiza tradicionalmente de forma empírica, donde los campesinos utilizan los residuos del tabaco (Nicotiana tabacum), conocido como tabaquina, para el control del ácaro de las aves Dermanyssus gallinae y en el control de garrapatas en bovinos en Camagüey.

Dentro del arsenal fitoterapéutico, destaca Morinda citrifolia L (noni) por sus múltiples propiedades medicinales atribuidas a sus frutos, raíz, corteza, semillas y, en particular, sus hojas, que poseen actividad 
antiinflamatoria, astringente, analgésica, antiséptica y antihelmíntica, entre otras (Salomón et al., 2009; Jiménez et al., 2012; Sánchez et al., 2012). Aunque esta es una es de las principales plantas utilizada en medicina complementaria (Ruíz et al., 2010), en Camagüey se le utiliza empíricamente para el control de garrapatas; no obstante, no existen estudios que validen su uso para este fin (Martínez et al., 2012).

Ante la necesidad de una comprobación científica de las bondades acaricidas de Morinda citrifolia L, el presente trabajo tuvo como objetivo la evaluación de su eficacia in vitro en comparación con productos químicos convencionales sobre teleoginas de Rhipicephalus (Boophilus) microplus.

\section{Materiales y Métodos}

\section{Localización del Estudio}

La investigación se realizó en la Unidad Básica de Producción Cooperativa «Fructuoso Rodríguez Pérez» del poblado Magarabomba, municipio «Carlos Manuel de Céspedes», provincia Camagüey, Cuba. En la zona se explotan bovinos mestizo cebú exentos de garrapaticidas químicos, que recibieron la vacunación contra Rhipicephalus microplus como refiere Vargas (2005). El experimento se realizó durante seis meses (septiembre de 2012 hasta febrero de 2013).

\section{Teleoginas}

Las teleoginas fueron colectadas al azar de 33 bovinos y trasladadas al laboratorio de parasitología de la Universidad de Camagüey «Ignacio Agramonte Loynaz». La clasificación e identificación taxonómica de $R$. microplus se hizo según Demedio y Meireles (1984).

Se descartaron las teleoginas que presentaron pérdidas de patas o palpos y aquellas con escaso movimiento. Se seleccionaron 240 garrapatas, colocándose 10 de ellas en cada placa Petri. Las garrapatas se lavaron con una solución de hipoclorito de sodio al $2.5 \%$ para prevenir la contaminación microbiana. Las garrapatas se fijaron con bandas de cinta adhesiva para evitar su escape y las 24 placas Petri se recubrieron con una malla porosa y se conservaron a temperatura ambiente.

\section{Extracto de Morinda citrifolia $\mathbf{L}$}

Se separaron hojas verdes de $M$. citrifolia L del resto de la planta y se secaron a la sombra durante 20 días, removiéndolas con frecuencia para evitar el desarrollo de hongos.

La obtención del extracto a partir de las hojas se hizo con etanol, según la Norma Ramal MINSAP 311/92. A los 21 días se procedió a la obtención del macerado con etanol en ciclos de tres extracciones cada cinco días. Se prepararon soluciones hidroalcohólicas en concentraciones de 10, 5, 2.5, 1.25 y $0.625 \%$, según lo referido por Chagas (A. Chagas, Brasil, Comunicación personal).

\section{Diseño Experimental}

Se formaron ocho tratamientos con tres réplicas por tratamiento, distribuidos de la siguiente manera:

T1: Extracto de M. citrifolia $\mathrm{L}$ al $10 \%$

T2: Extracto de M. citrifolia L al 5\%

T3: Extracto de M. citrifolia L al $2.5 \%$

T4: Extracto de M. citrifolia L al $1.25 \%$

T5: Extracto de $M$. citrifolia L al $0.625 \%$

T6: Control positivo (Supatraz ${ }^{\circledR} /$ amitraz) $1: 1000$

T7: Control positivo (Ticomín $囚 /$ cipermetrina) $1: 1000$

T8: Control negativo (agua destilada)

En el caso de los productos químicos Supatraz ${ }^{\circledR}$ y Ticomín ${ }^{\circledR}$ (IvoryChem, Singapur), se tuvo en consideración las recomendaciones del fabricante para la aplicación del esquema de tratamiento. 
En cada placa Petri se agregó $15 \mathrm{ml}$ de las concentraciones y productos acaricidas correspondientes a cada tratamiento por espacio de 5 minutos. Luego se procedió al retiro del producto utilizando papel absorbente.

\section{Interpretación de los Resultados}

Las respuestas a la acción de los tratamientos in vitro se realizaron a los 15 minutos y 24 horas posexposición. Se consideró la muerte de las teleoginas cuando no respondían al estímulo de la fuente luminosa de la lámpara halógena (estereoscopio) de $6 \mathrm{v}, 25$ W. Se tuvo como criterio la ausencia de reacción al momento de la palpación en la región cervical con un puntero romo y al no presentar movimientos de las patas o desplazamientos, según la metodología sugerida por Chagas (2008).

Se consideró como un resultado eficaz cuando se alcanza $60 \%$ de mortalidad como mínimo (Holsworth et al., 2006). En el cálculo de la eficacia, se utilizó la fórmula establecida por Zajac y Conboy (2006): (N. ${ }^{\circ}$ teleoginas vivas en el control negativo $-\mathrm{N} .^{\circ}$ teleoginas vivas en el tratamiento)/ N. ${ }^{\circ}$ teleoginas vivas en el control negativo), expresado en porcentaje.

Se realizó una comparación de proporciones de las teleoginas muertas entre los dos tiempos de lecturas por tratamientos y entre tratamientos con el apoyo del programa estadístico COMPROP 1 v. 2.1.

\section{Resultados y Discusión}

Se encontró una mayor proporción de teologinas muertas a las $24 \mathrm{~h}$ del tratamiento en relación con la lectura a los 15 min en los controles positivos con el uso de acaricidas químicos $(\mathrm{p}<0.01)$. No hubo diferencias estadísticas entre lecturas en los tratamientos con M. citrifolia L (Cuadro 1).
La diferencia en el número de teologinas muertas entre lecturas en los tratamientos 6 y 7 se explica por el efecto residual del producto acaricida. No obstante, la proporción de teologinas muertas fue menor que el reportado por Chagas (2008). La nula diferencia entre tiempos de lectura en los tratamientos con M. citrifolia L (T1 a T5) pudo deberse al uso de concentraciones muy bajas. Concentraciones mayores serán empleadas en estudios posteriores.

La mejor respuesta en número de garrapatas muertas se observó en T6, tanto a los 15 min como a las $24 \mathrm{~h}$ de la exposición

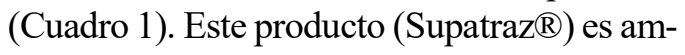
pliamente usado en Cuba, pero es importado, lo que encarece su uso, y está sujeto a la disponibilidad en el mercado internacional. En tal sentido, Polanco (2001) refiere que la disponibilidad de productos químicos para el control de garrapatas es cada vez menor debido a los altos costos, impacto ambiental y a la afectación en la inocuidad de la leche y carne.

El tratamiento con Supatraz $\mathbb{\circledR} /$ amitraz fue el más eficaz y superior a las concentraciones de M. citrifolia L (Figura 1). La baja proporción de muertes de teleoginas en los tratamientos que recibieron las distintas concentraciones de $M$. citrifolia L en el presente trabajo fueron, no obstante, superiores a los obtenidos por Peláez (2012) en su estudio alternativo con $N$. tabacum.

Mangold et al. (2000) y Guglielmone et al. (2007) destacan la importancia en la búsqueda de nuevos químicos y productos alternativos para el control eficaz de las poblaciones de garrapatas en las instalaciones pecuarias, con la finalidad de disminuir las pérdidas por concepto de carne y leche. Asimismo, la búsqueda de alternativas con productos económicos y de baja toxicidad deben abocarse a los métodos para la obtención del extracto de la planta, las mejores concentraciones y la forma de administración del producto (Chagas y Vieira, 2007; Shmahl et al. 2010; Giglioti et al., 2011). 
Cuadro 1. Proporción de teleoginas muertas a los 15 minutos y 24 horas $^{1}$ del tratamiento acaricida (30 garrapatas por tratamiento y por tiempo)

\begin{tabular}{lcccc}
\hline \multirow{2}{*}{ Tratamientos } & \multicolumn{2}{c}{ 15 minutos } & \multicolumn{2}{c}{ 24 horas } \\
\cline { 2 - 5 } & Total & Porcentaje & Total & Porcentaje \\
\hline T1 M. citrifolia L (10\%) & 2 & 6.7 & 5 & 16.6 \\
T2 M. citrifolia L (5\%) & 2 & 6.7 & 4 & 13.3 \\
T3 M. citrifolia L (2.5\%) & 1 & 3.3 & 3 & 10.0 \\
T4 M. citrifolia L (1.25\%) & 1 & 3.3 & 1 & 3.3 \\
T5 M. citrifolia L (0.625\%) & 0 & 0 & 1 & 3.3 \\
T6 (Supatraz $/$ amitraz) & 6 & $20.0^{\mathrm{a}}$ & 21 & $70.0^{\mathrm{b}}$ \\
T7 (Ticomín®/cipermetrina) & 4 & $13.3^{\mathrm{a}}$ & 9 & $30.0^{\mathrm{b}}$ \\
T8 (Control negativo) & 0 & 0 & 1 & 3.3 \\
\hline
\end{tabular}

${ }^{1}$ Los datos de garrapatas muertas a los $15 \mathrm{~m}$ y 24 horas son independientes (las muertes a los 15 min no se encuentran en el número de muertes a las $24 \mathrm{~h}$ )

${ }^{a, b}$ Superíndices diferentes entre tiempos y dentro de tratamientos indican diferencia estadística $(p<0.01)$

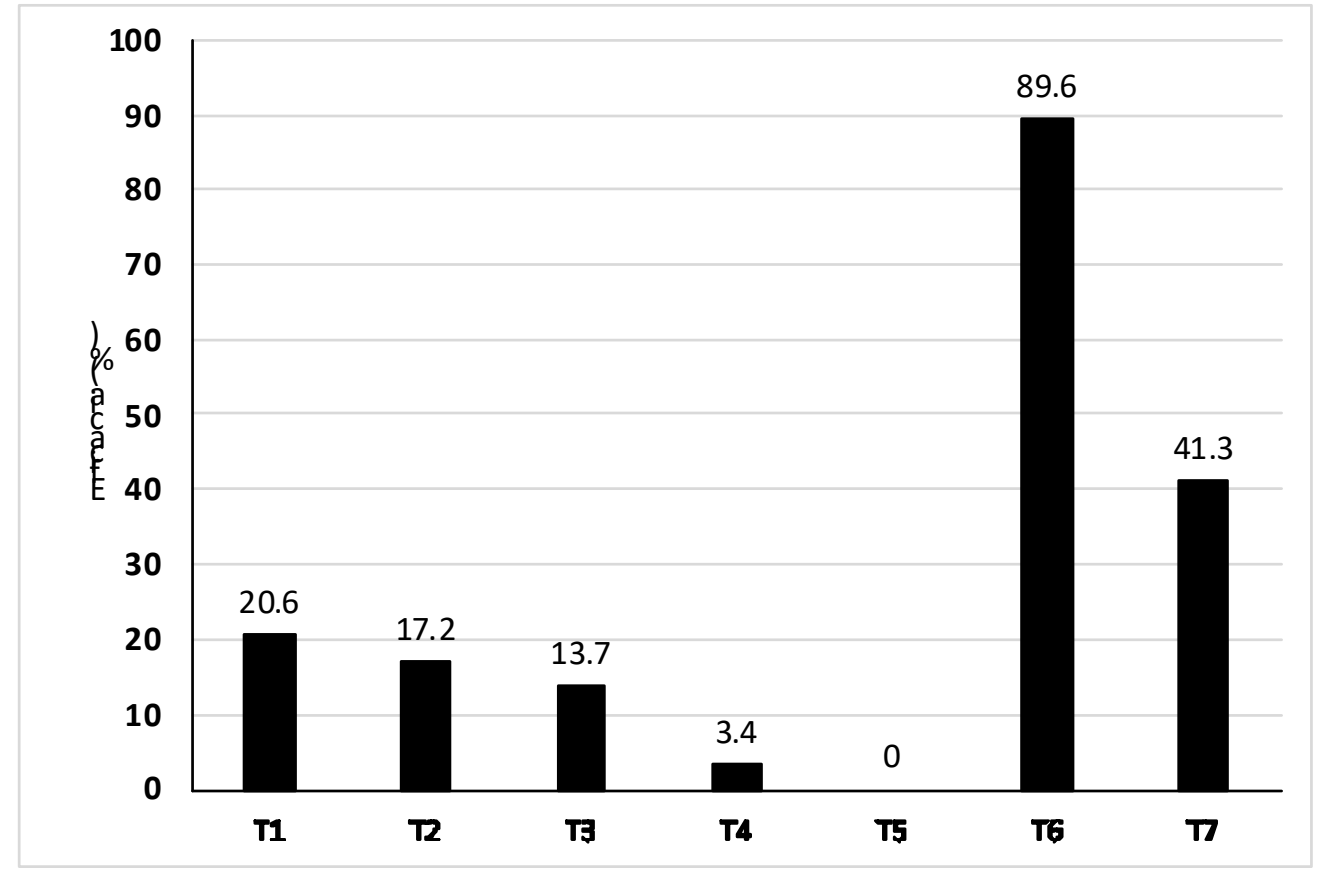

Figura 1. Eficacia de lo tratamientos con soluciones hidroalcohólicas de Morinda citrifolia L y de dos acaricidas convencionales sobre teleoginas Rhipicephalus microplus (T1 a

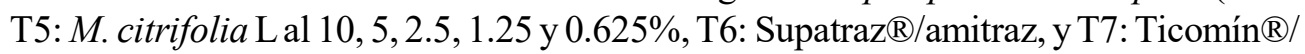
cipermetrina 


\section{Conclusiones}

No se pudo demostrar la eficacia de Morinda citrifolia $\mathrm{L}$ en las concentraciones utilizadas.

\section{Literatura Citada}

1. Alonso MA, Rodríguez RI, Fragoso H, Rosario R. 2006. Resistencia de la garrapata Boophilus microplus a los ixodicidas. Arch Med Vet 38: 105-113.

2. Bermúdez A, Velásquez E. 2002. Etnobotánica médica de una comunidad campesina del estado de Trujillo, Venezuela: un estudio preliminar usando técnicas cuantitativas. Rev Fac Farm 44: 2-6.

3. Chagas ACS, Vieira LS. 2007. Ação de Azadirachta indica (Neem) em nematódeos gastrintestinais de caprinos. Braz J Vet Res Anim Sci 44: 49-55.

4. Chagas ACS. 2008. Metodologías in vitro para avaliação de fitoterápicos e resultados de testes a campo. En: Congreso Brasileiro de Parasitologia Veternária. Curitiba, Brasil.

5. Demedio J, Meireles T. 1984. Manual de prácticas de parasitología I. La Habana: Ed EMPES. $124 \mathrm{p}$.

6. Estrada A, Venzal M. 2006. Highresolution predictive mapping for Boophilus annulatus and Boophilus microplus (Acari: Ixodidae) in Mexico and Southern Texas. Vet Parasitol 142: 350-358. doi: 10.1016/j.vetpar. 2006. 07.003

7. [FAO] Organización de las Naciones Unidas para la Alimentación y la Agricultura. 2003. Resistencia a los antiparasitarios: estado actual con énfasis en América Latina. Roma: FAO. [Internet]. Disponible en: http://www. fao.org/docrep/006/y4813s/ $\mathrm{y} 4813 \mathrm{~s} 03 . \mathrm{htm}$

8. Fernández FF, Freitas EPS. 2007. Acaricidal activity of an oleoresinous extract from Copaifera reticulata (Leguminosae: Caesalpinioideae) against larvae of the southern cattle tick, Rhipicephalus (Boophilus) microplus (Acari: Ixodidae). Vet Parasitol 147: 150-154. doi: 10.1016/j.vetpar.2007. 02.035

9. Giglioti R, Forim MR, Oliveira $H N$, Chagas ACS, Ferrezini J, Brito LG, et al. 2011. In vitro acaricidal activity of neem (Azadirachta indica) seed extracts with know azadirachtin concentrations against Rhipicephalus microplus. Vet Parasitol 181: 309-315. doi: 10.1016/j.vetpar.2011.03.053

10. Gil OR, Carmona AJ, Rodríguez MC. 2006. Estudio etnobotánico de especies tóxicas, ornamentales y medicinales de uso popular, presentes en el Jardín de Plantas Medicinales «Dr. Luis Ruiz Terán» de la Facultad de Farmacia y Bioanálisis de la Universidad de Los Andes. Bol Antropológico 24: 463-481.

11. Guglielmone AA, Castelli ME, Mangold AJ, Aguirre DH, Alcaraz E, Cafrune MM, Cetrá B, et al. 2007. El uso de acaricidas para el control de Rhipicephalus (Boophilus) microplus (Canestrini) (Acari: Ixodidae) en la Argentina. Rev Inv Agropec 36: 155-167.

12. Holsworth PA, Kemp D, Green P, Peter RJ, De Bruin C, Jonsson NN, Letonja T, et al. 2006. World Association for the Advancement of Veterinary Parasitology (W.A.A.V.P.) guidelines for evaluating the efficacy of acaricides against ticks (Ixodidae) on ruminants. Vet Parasitol 136: 29-43. doi: 10.1016/j.vetpar.2005.11.011

13. Jiménez MC, Martínez SM, Maceira MA, Pérez JL, Curi MA, Pérez H. 2012. Efecto del Noni-C sobre el peso corporal y los parámetros sanguíneos. Rev Cubana Plant Med 17: 321-329.

14. Mangold AJ, Muñoz Cobeñas ME, Castelli MC, Scherling NJ, Delfino MR, Guglielmone AA. 2000. Resistencia a la cipermetrina en una población de Boophilus microplus (Acari: Ixodidae) del norte de Santa Fe, Argentina. Rev Med Vet (Bs As) 81: 259-261. 
15. Martínez SM, Jiménez MC, Del Río $S$, Pérez JL, Maceira MA, Morales Z, Curi MA. 2012. Evaluación diurética del producto natural Noni-C (Morinda citrifolia L.) en un modelo experimental en ratas. Rev Cubana Plant Med 17: 431-438.

16. Ojeda MM, Rodríguez RI, Galindo E, Lezama R, Cruz C. 2011. Control de Rhipicephalus microplus (Acari: Ixodidae) mediante el uso del hongo entomopatógeno Metarhizium anisopliae (Hypocreales: Clavicipi-taceae). Revisión. Rev Mex Cienc Pec 2: 177-192.

17. Peláez M. 2012. Eficacia in vitro de la tabaquina en el manejo sostenible de Rhipicephalus microplus. Trabajo de Diploma. Cuba: Universidad de Camagüey Ignacio Agramonte y Loynaz. $57 \mathrm{p}$.

18. Polanco HC. 2001. Evaluación de un programa de control integrado contra la garrapata $B$. microplus en zonas lecheras de Villa Clara. Tesis de Magíster. La Habana, Cuba: Universidad Agraria de la Habana. 82 p.

19. Rodríguez RI, Alonso MA, Rodríguez F, Fragoso H, Santamaria VM, Rosario R. 2006a. Prevalence and potential risk factors for organophosphate and pyrethroid resistance in Boophilus microplus ticks on cattle ranches from the State of Yucatán, México. Vet Parasitol 136: 335-342.

20. Rodríguez RI, Rodríguez F, Alonso MA, Fragoso H, Santamaría VM, Rosario R. 2006b. Prevalence and potential risk factors for amitraz resistance in Boophilus microplus ticks in cattle farms in the State of Yucatan, Mexico. Prev Vet Med 75: 280-286.

21. Rodríguez RI, Arieta JR, Pérez LC, Rosado JA, Ramírez GT, Basto-Estrella $\boldsymbol{G}$ 2010. Uso de lactonas macrocíclicas para el control de la garrapata Rhipicephalus (Boophilus) microplus en el ganado bovino. Arch Med Vet 42: 115-123. doi: 10.4067/S0301-732X20 10000300002

22. Rodríguez, RI, Quiñones AF, Fragoso $S H .2005$. Epidemiología y control de la garrapata Boophilus en México. En:
Rodríguez RI (ed.). Enfermedades de importancia económica en producción animal. México DF: McGraw-HillUADY. p 571-592.

23. Rodríguez RI, Rivas AL, Chowell G, Fragoso SH, Rosario CR, García Z, Smith SD, et al. 2007. Spatial distribution of acaricide profiles (Boophilus microplus strains susceptible or resistant to acaricides) in south eastern Mexico. Vet Parasitol 146: 158-169.

24. Ruíz SG, Venegas EA, Chávez H, Eustaquio CL. 2010. Identificación preliminar de los metabolitos secundarios de los extractos acuosos y etanólicos del fruto y hojas de Morinda citrifolia L «noni» y cuantificación espectrofotométrica de los flavonoides totales. UCV Scientia 2(2): 11-22.

25. Salomón S, López OD, García CM, González ML, Fusté V. 2009. Desarrollo de una tecnología para la obtención de extracto acuoso de hojas de Morinda citrifolia L (noni). Rev Cubana Plant Med 14(2): 1-8.

26. Sánchez $N, B u M$, Pérez-Saad $H$, Lara G, Scull I. 2012. Efecto del zumo de Morinda citrifolia L (noni) en modelos de analgesia. Rev Cubana Plant Med 17: 330-337.

27. Schmahl G, Al-Rasheid K, AbdelGhaffar F, Klimpel S, Mehlhorn $H$. 2010. The efficacy of neem seed extracts (Tre-san ${ }^{\circledR}$, MiteStop ${ }^{\circledR}$ ) on a broad spectrum of pests and parasites. Parasitol Res 107: 261-269. doi: 10.1007/ s00436-010-1915-x

28. Taylor M, Coop MA, Wall RL. 2007. Veterinary parasitology. $3^{\text {rd }}$ ed. London, UK: Blackwell. $482 \mathrm{p}$.

29. Vargas M, Montero C, Sánchez D, Pérez D, Joglar M, Machado H, Oliva $R$, et al. 2005. La aplicación de una dosis de refuerzo con el inmunógeno Gavac Plus es suficiente para estimular un nivel efectivo de inmunidad en rebaños con más de un año sin inmunización. En: Congreso Biotecnología. La Habana.

30. Zajac AM, Conboy GA. 2006. Veterinary clinical parasitology. $7^{\text {th }} \mathrm{ed}$. 320: Wiley-Blackwell. 320 p. 